\title{
Timing properties of gamma-ray bursts detected by SPI-ACS detector onboard of INTEGRAL
}

\author{
V. Savchenko* \\ François Arago Centre, APC, Université Paris Diderot, CNRS/IN2P3, CEA/Irfu, Observatoire de \\ Paris, Sorbonne Paris Cité, 13 rue Watt, 75013 Paris, France \\ E-mail: Volodymyr.Savchenko@unige.ch
}

\section{A. Neronov}

ISDC Data Centre for Astrophysics, Ch. d'Ecogia 16, 1290 Versoix, Switzerland, Observatory of Geneve, University of Geneva, Ch. des Maillettes 51, 1290 Sauverny, Switzerland

E-mail: Andrii.Neronov@unige.ch

\section{T.J.-L. Courvoisier}

ISDC Data Centre for Astrophysics, Ch. d'Ecogia 16, 1290 Versoix, Switzerland, Observatory of Geneve, University of Geneva, Ch. des Maillettes 51, 1290 Sauverny, Switzerland

E-mail: Thierry.Courvoisier@unige.ch

We study timing properties of a large sample of $\gamma$-ray bursts (GRB) detected by the AntiCoincidence Shield (ACS) of the SPI spectrometer of INTEGRAL telescope. First we identify GRB-like events in the SPI-ACS data. The data set under investigation is the history of count rate of the SPI-ACS detector recorded with a binning of $50 \mathrm{~ms}$ over the time span of $\sim 10 \mathrm{yr}$. In spite of the fact that SPI-ACS does not have imaging capability, it provides high statistics signal for each GRB event, because of its large effective area. We classify all isolated excesses in the SPI-ACS count rate into three types: short spikes produced by cosmic rays, GRBs and Solar flare induced events. We find some $\sim 1500$ GRB-like events in the $10 \mathrm{yr}$ exposure. A significant fraction of the GRB-like events identified in SPI-ACS occur in coincidence with triggers of other gamma-ray telescopes and could be considered as confirmed GRBs. We study the distribution of durations of the GRBs detected by SPI-ACS and find that the peak of the distribution of long GRBs is at $\simeq 20$ s, i.e. somewhat shorter than for the long GRBs detected by BATSE. Contrary to the BATSE observation, the population of short GRBs does not have any characteristic time scale. Instead, the distribution of durations extends as a powerlaw to the shortest time scale accessible for SPIACS,$\leq 50 \mathrm{~ms}$. We also find that a large fraction of long GRBs has a characteristic variability time scale of the order of $1 \mathrm{~s}$. We discuss the possible origin of this time scale. Finally, we report recent SPI-ACS detection of unusual unexplained bursting activity, unlike any other observed by SPI-ACS in 10 years of operation. We discuss possible origin of this activity.

An INTEGRAL view of the high-energy sky (the first 10 years) - 9th INTEGRAL Workshop and celebration of the 10th anniversary of the launch

15-19 October 2012

Bibliotheque Nationale de France, Paris, France

\footnotetext{
*Speaker.
} 


\section{Introduction}

Gamma-ray bursts (GRBs) are the most violent explosions in the universe. In spite of the long observation history, the nature of the GRB phenomenon remains obscure. Isotropy of spatial distribution (15) and spectral features in long-lasting afterglows discovered by Beppo/SAX (16) confirmed the cosmological nature of GRB phenomenon. Up to now, the largest sample of GRBs available for a statistical study has been collected by the CGRO/BATSE (15) and Konus-Wind (1). One of the main findings of BATSE and Konus-Wind was the discovery of a dichotomy in the distribution of GRB durations $(12 ; 10)$. This distribution reveals two broad bumps separated roughly at $T_{90} \simeq 2 \mathrm{~s}(10 ; 15)$. This dichotomy is commonly believed to be related to the difference in burst progenitors. The long GRBs seem to be associated with gravitational collapse of the cores of massive stars (11). The short GRBs are possibly related to coalescence of compact objects (2).

The focus of the present study is on the timing properties of GRBs detected by the AntiCoincidence Shield (ACS) of spectrometer SPI on board of the INTEGRAL sattellite (24). Although SPI-ACS does not have an imaging capability, it has exceptionally large effective area (up to about $1 \mathrm{~m}^{2}$ ). SPI-ACS readout does not rely on any on-board trigger criteria and instead continuously measures the event rate all over the INTEGRAL mission lifetime with a time binning of $50 \mathrm{~ms}$. The first attempt to systematically study GRBs with SPI-ACS was done by Rau et al. (17) using about 800 days of data and identifying 179 GRBs also detected by other satellites, but visible in the SPI-ACS. Considerably more data has been collected since the first catalogue and an advance in understanding the systematic effects suggested the new study of the SPI-ACS burst population.

\section{Detection of GRBs}

We use the SPI-ACS data of 1030 revolutions of the INTEGRAL satellites, from 20 to 1100 ((3230 days from 2002-12-12 to 2011-10-16)). Data quality criteria restrict the usable exposure time to 2412 days. We identify burst-like events on 10 different time scales, from $100 \mathrm{~ms}$ to $30 \mathrm{~s}$. The local background count rate is estimated on larger time scales up to $10^{4} \mathrm{~s}$. Excess variance of the background count rate is used to correct significance of the detection. Regions with exceedingly high excess variance are exluded from the analysis. The total number of burst-like events found in our analysis is 82231. Almost all these events are so-called "short spikes" (17). At long time scales, variations of the SPI-ACS count rate are mostly due to the Solar activity. Most of the bursts of solar origin can be excluded by studing stability of the background. We also use solar weather prediciton center (SWPC) reports ${ }^{1}$ to find affected time intervals.

\section{Short spikes}

The majority of burst-like events (about 30 spikes/day (17)) in SPI-ACS are "short spikes", with typical durations of about the about $50-100 \mathrm{~ms}$, i.e. one or two bins of the lightcurve. The nature of these events was controversial. The analysis by Rau et al. (17) suggested that the spikes are produced following hits of the ACS by high-energy cosmic rays (protons with the energy of $\sim 30 \mathrm{TeV}$ or heave nuclei of $\sim 2 \mathrm{GeV}$ ). Deluit et al. (6) have noticed that all the particles from a

1. http ://www.swpc.noaa.gov/ 


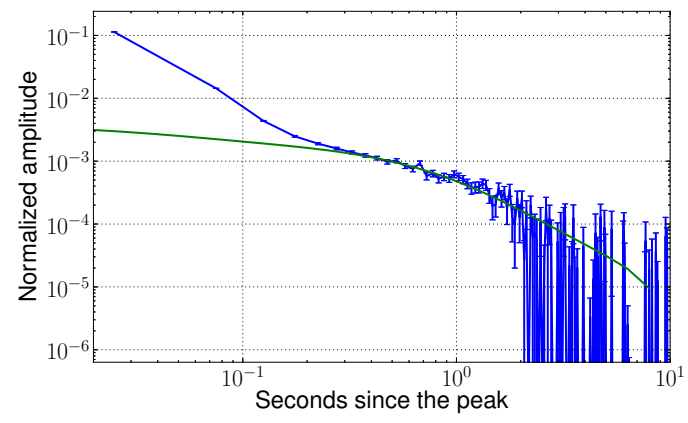

FIGURE 1: The stacked profile of the spikes (blue data points). The extended tail is fitted with the decay of residual cosmic ray induced radioactivity of the BGO found from FLUKA simulations (green curve).

cosmic ray induced shower in ACS would be counted as just one particle. They also could be real hard GRBs escaping detection by other instruments (5).

The properties of the short spikes reveal remarkable stability over the whole mission span (10 years of data). It means that they are associated with high energy protons. The stacking analysis reveals weak $(0.1 \%)$ afterglow lasting for at least 3 seconds (Figure 1$)$. The profiles of the brightest individual spikes follow closely the averaged stacked profile. The shower particles certainly could not be responsible for the second-long afterglow shown in Fig. 1. Instead, the tail could be attributed to short-term activation of the BGO crystals with the cosmic ray protons. We have investigated the radioactivity induced in the BGO crystals by high-energy cosmic ray passage using FLUKA Monte-Carlo code $(3 ; 8)$ and found that it fits the data well.

All the spikes are expected to have identical time profiles. Template-based rejecting removes most of the spikes, suppressing only a small fraction of the real GRBs.

\section{GRBs detected by SPI-ACS}

Rejection of spikes and Solar flare events from the overall sample of burst-like events in SPIACS leaves a sample of 1416 GRB lightcurves available for the timing analysis. Figure 2 shows the distribution of durations $T_{90}$ for these GRBs. The black solid histogram shows the distribution of durations of events occurring in coincidence with the triggers from other detectors. It is consistent with that of the independent candidate sample.

Our analysis of the sample of GRBs detected by SPI-ACS shows that the distribution of GRB durations shown in Figure 2 has two components. But contrary to BATSE burst duration distribution, the distribution of durations of SPI-ACS bursts does not reveal short bursts concentrated around a characteristic time scale. Instead, the burst duration distribution follows a powerlaw at short time scales $T_{90} \lesssim 3 \mathrm{~s}$ extending to the shortest time scale accessible to the ACS. It suggests that the difference between the short and the long bursts may not be fully characterized by the commonly accepted criterion $T_{90} \lessgtr 2 \mathrm{~s}$. Very short bursts are confused with the cosmic ray induced spikes in ACS. 


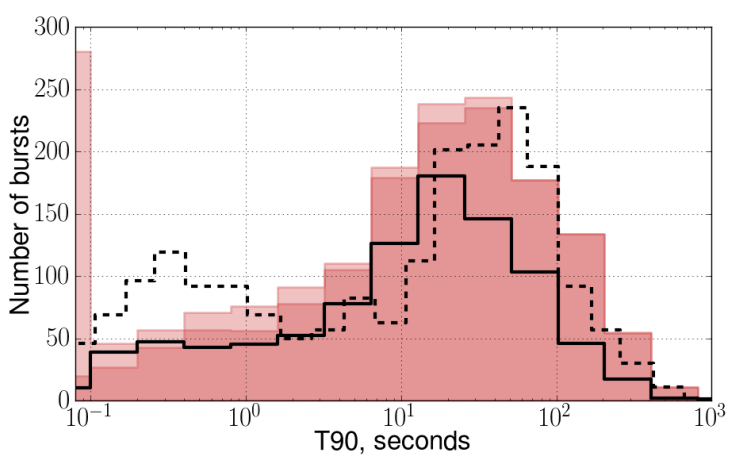

FIgURE 2: Distributions of the $T_{90}$ durations. Dark and light shadings of the filled region correspond to 5 and 3 sigma confidence level for the event not being a spike. Solid black curve is for the confirmed bursts. The distribution of durations of GRBs from BATSE 4B GRB sample, scaled to match the normalization of the ACS sample, is shown by the black dashed line.

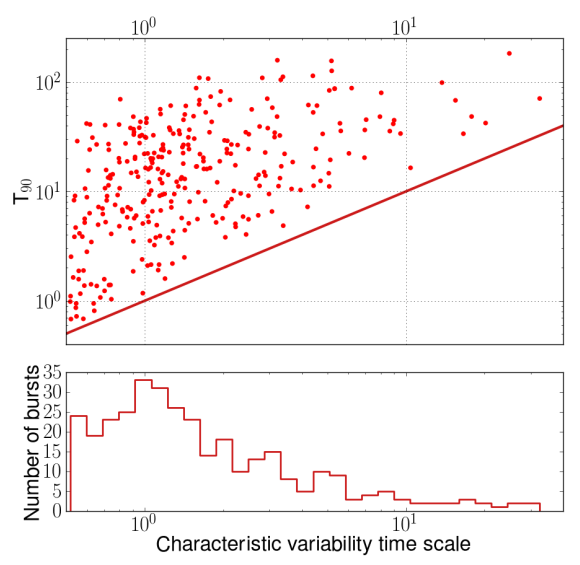

Figure 3: One the top panel : the first characteristic time scale vs duration $\left(T_{90}\right)$ for long GRBs. On the bottom panel : the distribution of the first characteristic time scales.

Next, we use the first order structure function to to identify a characteristic variability time scale. Figure 3 shows the characteristic time scales of long GRBs as a function of the GRB duration. One could see that large fraction of GRBs has the characteristic time scale $T_{v a r}=1 \pm 1 \mathrm{~s}$. This analysis confirms the result of (4). This time scale is related to typical separation between the individual pulses or activity periods of GRBs. Interestingly, it coinsides with the time scale at which a break in the distribution of GRB durations is observed. The distinct characteristic time scale of the order of a second might be associated with the variability of the GRB central engine. Alternatively, it can be related to the size of the emission region. Further investigation of the nature of the characteristic time scale, exploiting the rich timing data of the SPI-ACS, is required to clarify its origin.

\section{Mysterious bursts of April-May 2012}

From of April 19th 2012 to April 21st, SPI-ACS detected 25 mostly short (from 50 ms but few reaching $8 \mathrm{~s}$ ) bursts at a significance level of 8-10 sigma (with the peak count-rate of $10^{4}$ $10^{5}$ counts/s on $50 \mathrm{~ms}$ time bin). Other 7 bursts were detected from May 5th to 7th (Atel 4101). The highest peak count-rate recorded by the ACS was $1.5 \times 10^{5}$ counts/s (detection significance up to 50 sigma), suggesting fluences up to $3.5 \times 10^{-6} \mathrm{erg} / \mathrm{cm}^{2} \mathrm{~s}$ above $75 \mathrm{keV}$. Light curves of these bursts resemble strongly those of a magnetar outburst (sometimes observed also by SPI-ACS (19)) As no spatial information are available from the ACS, this activity could not be firmly associated this activity with any known source.

None of these events have not been reported by other GRB monitoring experiments. In the previous sections we described thorough study of properties of the SPI-ACS light curve (see also (20)). It allows us to claim that no instrumental issue that could give rise to similar effects was ever 
observed. Assuming these bursts are real astrophysical events, one has to explain how they avoided detection by all other gamma-ray detectors but SPI-ACS.

The first possibility is in the visibility constrains. For the near-Earth satellites (e.g. Fermi or Suzaku) half of the sky is always occulted. It is straightforward to estimate that for some position of the source only one (but also at least one) relatively weak burst would be visisble for the Fermi/GBM. On the other hand, Konus-Wind is positioned in L1 point and does not have any visibility limitations.

Secondly, unlike SPI-ACS, most of the GRB detectors rely on an on-board trigger algorithm to choose the data aquisition mode. Unless the trigger condition is satisfied, the recorded data is usually relatively limited, suitable for studying only bright bursts. This may be the case for Suzaku/WAM and Konus-Wind. However, the offline data of Fermi/GBM is quite detailed - it includes a light curve with 64 ms resolution - similar to the one recorded by SPI-ACS.

Finally, the bursts may be simply below sensitivity for every instrument but SPI-ACS. It appears to be not impossible, as SPI-ACS possesses exceptionally large effective area and is especially sensitive above $\sim 1 \mathrm{MeV}$ - where many other instruments usually run out of statistics. To provide sufficient flux at $1 \mathrm{MeV}$ to be visible in SPI-ACS but fall below the sensitivity of Fermi/GBM, Swift/BAT and Konus-Wind (all a lot more sensitive at $10-1000 \mathrm{keV}$ then SPI-ACS), the bursts would have to feature ususually hard spectrum.

To investigate the possible spectra of the bursts we simultaneously fitted the to Fermi/GBM and SPI-ACS spectra. The SPI-ACS spectra consisted in fact of a single bin. The corresponding response was modeled by a simple shape, resembling the results of Monte-Carlo simulations. The energy and angular dependence of the response was validated by cross-calibration on known bright GRBs.

As expected, OTTB spectrum can be firmly excluded with the significance close to the detection significance of the given (the "minimal" - see above) burst in SPI-ACS - about 8 sigma. For the broken powerlaw "Band" model or thermal spectrum peaking at $1 \mathrm{MeV}$ the spectra are marginally consistent. Black body spectrum peaking close to the edge of the GBM energy range, at $100 \mathrm{MeV}$ is not formally excluded. So unusually hard spectrum would provide strong signal in the energy range of Fermi/LAT. LAT sky exposure converage during the duration of the 25 bursts is small, less then a third of the sky is sufficiently exposed. However, it is likely that thousands of photons above $100 \mathrm{MeV}$ would be seen by LAT even from out of the field of view.

Another remaining possiblily is that the bursts were caused a peculiar and unexplained instrumental effect. Even if this is the case, finding an explanation for this effect would be very interesting.

Usefullness of the GBM for confirming or rejecting bursts detected by SPI-ACS is mainly limited by observational constrains. Using the data of the Konus-Wind instrument, not affected by occultations, although limited by the trigger criteria, might prove to be useful to finally clarify the nature of these mysterious bursts events.

\section{Références}

[1] Aptekar, R. L., Frederiks, D. D., Golenetskii, S. V., et al. 1995, Br, 71, 265

[2] Barthelmy, S. D., Chincarini, G., Burrows, D. N., et al. 2005, Nature, 438, 994 


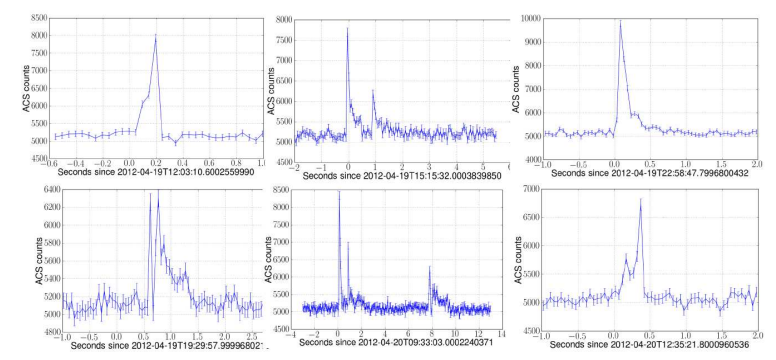

Figure 4: Some of the unidentified bursts detected by INTEGRAL/SPI-ACS between April 19th and May 7th 2012.

[3] Battistoni, G., Cerutti, F., Fassò, A., et al. 2007, in American Institute of Physics Conference Series, Vol. 896, Hadronic Shower Simulition Workshop, ed. M. A. . R. Raja, 31-49

[4] Beloborodov, A. M., Stern, B. E., \& Svensson, R. 2000, ApJ, 535, 158

[5] Cline, D. B., Matthey, C., Otwinowski, S., Czerny, B., \& Janiuk, A. 2007, in American Institute of Physics Conference Series, Vol. 921, The First GLAST Symposium, ed. S. Ritz, P. Michelson, \& C. A. Meegan, 280-283

[6] Deluit, S., Produit, N., Mowlavi, N., Rohlfs, R., \& Dubath, P. 2004, in ESA Special Publication, Vol. 552, 5th INTEGRAL Workshop on the INTEGRAL Universe, ed. . C. W. V. Schoenfelder, G. Lichti, 641

[7] Fenimore, E. E., in 't Zand, J. J. M., Norris, J. P., Bonnell, J. T., \& Nemiroff, R. J. 1995, ApJ, 448 ,

[8] Ferrari, A., Sala, P. R., Fassò, A., \& Ranft, J. 2005, FLUKA : A multi-particle transport code (program version 2005) (Geneva : CERN)

[9] Kommers, J. M., Lewin, W. H. G., Kouveliotou, C., et al. 1998, ArXiv Astrophysics e-prints

[10] Kouveliotou, C., Meegan, C. A., Fishman, G. J., et al. 1993, ApJ, 413,

[11] MacFadyen, A. I. \& Woosley, S. E. 1999, ApJ, 524, 262

[12] Mazets, E. P., Golenetskii, S. V., Ilinskii, V. N., et al. 1981, Ap\&SS, 80, 3

[13] Mereghetti, S., Götz, D., Weidenspointner, G., et al. 2009, ApJ, 696,

[14] Minaev, P. Y., Pozanenko, A. S., \& Loznikov, V. M. 2010, Extended Emission from Short Gamma-Ray Bursts Detected with SPI-ACS/INTEGRAL

[15] Paciesas, W. S., Meegan, C. A., Pendleton, G. N., et al. 1999, The Astrophysical Journal, 122, 465

[16] Piro, L., Amati, L., Antonelli, L. A., et al. 1998, å, 331,

[17] Rau, A., Kienlin, A. v., Hurley, K., \& Lichti, G. G. 2005, ASTRON.ASTROPHYS., 438, 1175 
[18] Sakamoto, T., Barthelmy, S. D., Baumgartner, W. H., et al. 2011, ApJS, 195, 2

[19] Savchenko, V., Neronov, A., Beckmann, V., Produit, N., \& Walter, R. 2010, A\&A, 510,

[20] Savchenko, V., Neronov, A., Courvoisier, T. J.-L., 2012, A\&A, 541, 122

[21] Scargle, J. D. 1998, ApJ, 504, 405

[22] Vedrenne, G., Roques, J. P., Schönfelder, V., et al. 2003, A\&A, 411,

[23] Vigano, D. \& Mereghetti, S. 2009, POS, extremesky, 049

[24] von Kienlin, A., Beckmann, V., Rau, A., et al. 2003, A\&A, 411,

[25] von Kienlin, A., Beckmann, V., Rau, A., et al. 2003, ApJ, 411,

[26] Winkler, C., Courvoisier, T. J. L., Di Cocco, G., et al. 2003, åp, 411,

[Atel 4101] Savchenko et al 2012, Atel 4101 